\title{
Museo, memoria y patrimonio cultural. Contexto de la independencia peruana, 1821-18261
}

Recibido: 21/08/2021

Aprobado: 28/1/2021

\author{
HEBNER CUADROS CHÁVEZ \\ Universidad Nacional Mayor de San Marcos (UNMSM) \\ hebner.cuadros@unmsm.edu.pe \\ https://orcid.org/0000-0002-8840-125X
}

\section{RESUMEN}

La valoración de la memoria histórica del Perú mediante el patrimonio cultural y su relevancia en el ideario político y en la propuesta discursiva, durante el preludio y desarrollo de la independencia, determinaron los primeros planteamientos de las políticas culturales en el proceso de construcción de la nueva república peruana. Estas condiciones afianzaron la voluntad política de la autoridad y el compromiso de crear el Museo Nacional del Perú como entidad de salvaguarda, educación y reconocimiento de la memoria histórico-cultural. La siguiente investigación presenta un análisis e interpretación de los factores sociales que contribuyeron en el valor del legado cultural como recurso simbólico, en un periodo histórico del Perú, y los efectos en su desarrollo.

Palabras clave: museo, memoria, patrimonio cultural, independencia peruana, bicentenario del Perú

\section{Museum, Memory and Cultural Heritage. Context of the Peruvian Independence, 1821-1826}

\begin{abstract}
The valuation of the historical memory of Peru through the cultural heritage and its relevance in the political ideology and in the discursive proposal, during the prelude and development of independence, determined the first proposals of cultural policies in the process of construction of the new Peruvian Republic. These conditions strengthened the political will of the authority, and the commitment to create the National Museum of Peru as an entity for safeguarding, education and recognition of historical-cultural memory. The present study offers an analysis and interpretation of the social factors that contributed to the value of the cultural legacy as a symbolic resource, in a historical period of Peru, and the effects on its development.
\end{abstract}

Keywords: museum, memory, cultural heritage, Peruvian independence, bicentennial of Peru

1 Este artículo es consecuencia investigativa del proceso de elaboración de la tesis de maestría El museo como equipamiento cultural metropolitano. Políticas de gestión, de la Maestría en Gestión del Patrimonio Cultural, Facultad de Ciencia Sociales de la Universidad Nacional Mayor de San Marcos (UNMSM). Así mismo, este artículo es parte de la investigación registrada en el VRIP-UNMSM con el código E19151321. Sus primeros hallazgos históricos y enfoques teóricos fueron planteados a nivel de ponencia académica en el IX Congreso Nacional de Historia. Lima 2020. Luego, se incorporó como producto de artículo científico en el Proyecto del Grupo de Investigación Historia y Ciudadanía Activa, de la Facultad de Ciencias Sociales-UNMSM. 


\section{Introducción}

I os temas de patrimonio cultural y la museística en el contexto de la independencia peruana — concerniente al recurso de identidad — aún son jescasos y requieren interpretaciones desde diversos enfoques investigativos para contribuir al análisis y comprensión del desarrollo cultural en el país, como parte de nuestra historia, memoria e identidad. El presente artículo, interpreta con amplitud el tema museístico en relación con el patrimonio cultural, las políticas culturales y su impacto con la reminiscencia social durante el proceso de la independencia del Perú. Inferimos que, en la actual coyuntura histórica, es importante repensar e interpretar los aspectos culturales, especialmente en el ámbito museístico y del patrimonio cultural, como proceso de implementación en torno a las políticas culturales del periodo de la independencia del Perú durante el quinquenio de 1821 a 1826.

El tema que aquí presentamos es relevante, porque se contextualiza durante el progreso de cambios hacia una nueva república, cuyos efectos condicionaron los procesos posteriores en la gestión cultural y en la propuesta de políticas culturales del Perú. Por consiguiente, las disquisiciones desarrolladas en el presente artículo tienen el propósito de contribuir al conocimiento de los aspectos culturales de inicios de la etapa republicana del país. Así mismo, buscan motivar al debate académico.

Como contribución investigativa, los planteamientos señalados en el artículo son aportes académicos sobre la historia de la museología y de las políticas culturales en el Perú, como también para la comprensión de la memoria histórica en la dimensión de identidad peruana. Así mismo, planteamos la siguiente hipótesis: la memoria histórica y el patrimonio cultural fueron referentes emblemáticos de la identidad nacional en el proceso de la independencia peruana. A partir de ello, aspiramos a crear un espacio de debate nacional en el contexto de la historia cultural peruana.

Con el desarrollo del artículo, nuestro objetivo general es explicar los aspectos de la memoria histórica, del patrimonio cultural y del componente museístico como ejes de identidad en el proceso de la independencia, e interpretar el uso y la valoración de dichos aspectos, como factor ideológico en el contexto de la emancipación.

Este trabajo se divide en tres partes. En el primer apartado desarrollamos las fundamentaciones conceptuales y las reflexiones sobre la memoria histórica, el patrimonio cultural, el museo y las políticas culturales del Perú 
en el proceso de la independencia, planteando interpretaciones en torno a la valoración de la memoria histórica. En el segundo segmento, determinamos las propuestas y condiciones del ideario político e ideológico en la etapa de la independencia, con base en el legado cultural y en la memoria histórica durante el proceso de cambio. Por último, analizamos los planteamientos de las políticas culturales en el proceso hacia una nueva república, que contribuyeron a la creación del Museo Nacional en el Perú.

\section{Fundamentos conceptuales y valoración de la memoria histórica}

Para comprender el significado del valor patrimonial de la historia y cultura, en el contexto social de la independencia, es primordial deducir las categorías conceptuales de cinco elementos esenciales: museo, memoria, patrimonio cultural, pasado y tradición. Estos han sido factores de aprehensión sobre los legados culturales de la nación, siempre presentes en el devenir de las sociedades e ineludibles a pesar de las transformaciones de la colectividad; han sido asumidas, según la idiosincrasia y coyuntura de los pueblos. Son factores determinantes, que conectan al individuo y a la sociedad con herencias culturales y su cosmovisión, lo cual les permite discernir sobre su historia y su cultura, así como establecer valores sobre ellos.

Como primera base conceptual, sustentamos que el «museo» es una entidad que alberga determinadas producciones y creaciones humanas, a condición de su protección, estudio y conocimiento, por los méritos que posee y los valores que representa. Esta enunciación expresa la esencia de la institución museística ${ }^{2}$ desde el momento de su establecimiento en el mundo como fenómeno cultural y, también, a partir de su primera fundación en el Perú. Con el tiempo, y según la experiencia de uso en las naciones, se sistematizaron nuevos conceptos consensuados, como lo planteado en la Convención Mundial del ICOM, en Viena en el 2007, estipulándose que el museo «es una institución [...] al servicio de la sociedad [...], que adquiere, conserva, estudia, expone y difunde el patrimonio material e inmaterial de la humanidad con

2 Fue la finalidad originaria del museo — que aún tiene ponderación en la mayoría de los museos peruanos- - pero en la medida de las demandas de servicio que le compete, y por consecuencia de su desarrollo como entidad, la naturaleza de las funciones de algunos museos en el Perú y el mundo tuvo cambios paulatinos desde inicios del siglo XX. En la actualidad, continúa con innovaciones, específicamente en la gestión de actividades culturales, académicas y científicas; así mismo, en el servicio social para un mejor valor de uso. Por esos cambios, se estableció la denominación de «museo vivo». 
fines de estudio, educación y recreo» (ICOM 2007, 3). Luego, se propuso un nuevo concepto en la Conferencia General del ICOM, en Kioto, en el marco de la Vigesimoquinta Convención de la UNESCO 2019, determinándose que:

Los museos son espacios democratizadores [...] para el diálogo crítico sobre los pasados y los futuros. [...] custodian artefactos y especímenes para la sociedad, salvaguardan memorias diversas para las generaciones futuras, y garantizan la igualdad de derechos y la igualdad de acceso al patrimonio para todos los pueblos.

Los museos [...] Son participativos y transparentes [...] a fin de coleccionar, preservar, investigar, interpretar, exponer, y ampliar las comprensiones del mundo, con el propósito de contribuir a la dignidad humana y a la justicia social, a la igualdad mundial [...] (ICOM 2019, 2).

Esta propuesta de definición no fue aprobada, ${ }^{3}$ quedando vigente - por consenso- el concepto anterior del 2007 hasta la actualidad. En esencia, dichos conceptos museísticos ratifican la importancia institucional del museo como vehículo para el reconocimiento cultural y fortalecimiento de la identidad social.

Por otra parte, la «memoria» es una categoría de evocación colectiva e individual, orientada al aspecto histórico y cultural que la sociedad conserva como tenencia identitaria y base de su desarrollo. Su esencia es el conocimiento de los hechos y las creaciones de la humanidad dentro de un contexto histórico-social, para ser conservado y tener continuación en el tiempo. La manifestación de la «memoria» se da mediante un proceso conectivo de las personas y su grupo social, en relación al pasado de su entorno, destacándose la valoración de lo evocado y la construcción de significados para darle sentido ${ }^{4}$ a la remembranza.

Así mismo, consideramos que el «patrimonio cultural» es la categoría designada a una entidad cultural, producto de la creación humana y con valores otorgados por la sociedad de acuerdo con sus representaciones. Estas trascienden por el valor de uso y el potencial simbólico que se construye sobre dicha entidad, ya que su naturaleza está relacionada con lo histórico como testimonio material o inmaterial de una civilización, cuyas significaciones son fluctuantes en el tiempo.

3 No hubo consenso, porque el concepto solo se centraba en la salvaguarda y la educación, y no recogía las nuevas tendencias del «museo activo», de servicio a los retos sociales de la actualidad, en su nivel de institucionalidad.

4 Referido al entendimiento y comprensión de la memoria colectiva. 
Respecto al concepto de «pasado», consideramos que culturalmente es el «tiempo y la herencia» para el autorreconocimiento social y su continuidad. Son vestigios de bienes que merecen conservarse según los intereses y formas de organización de la sociedad, desarrollándose valores y sentimientos en torno a ella, en busca de una identidad. Según la teoría de Ballart (2002, 38-39), lo «pasado» se personifica mediante el patrimonio cultural y se enarbola con significados: «[...] lo único seguro con que cuenta el ser humano es con su pasado, puesto que de allí proviene. Esta válvula de seguridad funciona desde el momento en que el hombre utiliza la facultad de recordar».

Otra base conceptual es la «tradición», que se consolidada mediante la comprensión de un bien cultural, y sobre el cual se genera un valor simbólico desde la percepción colectiva o individual, cuya perdurabilidad es garantizada por el valor de uso. Dependiendo de su contexto, puede permanecer en la memoria colectiva o simplemente extinguirse. En este proceso son determinantes las categorías y tipologías del bien cultural o «capital cultural simbólico» que se configura como habitus, según la teoría de Pierre Bourdieu (1990), y por su usanza se empodera para los intereses de la sociedad o de un sector de ella.

La memoria histórica-cultural está sujeta a condiciones sociales, ideológicas y políticas, porque en un caso se puede tener identificación plena con un bien patrimonial o hecho histórico; en otro, solo se manifiesta la admiración por un afán de curiosidad. Así mismo, para algunos, la memoria se mantiene por un hábito social y cultural de conservar una tradición en el sentido de nostalgia. En estos casos, las formas de reminiscencia generan preferencias de valoración y significación hacia el objeto u hecho histórico.

En el Perú, el ambiente cultural entre el periodo de inicio y consolidación de la independencia mostró un sentimiento de admiración e interés de estudio y reconocimiento hacia las raíces históricas y civilizaciones originarias, especialmente a la cultura inca. Fue un proceso idiosincrático, que venía consolidándose progresivamente desde las décadas del preludio a la independencia peruana, impulsándose ideas reivindicatorias hacia la herencia histórica y a las manifestaciones culturales del Perú. Los cambios de pensamiento estaban alojados — principalmente — en los círculos académicos progresistas, influenciados por los aportes científicos interdisciplinarios ${ }^{5}$ desde mediados del siglo

5 Por un lado, se encargaban estudios dedicados a las ciencias naturales y a la minería, pero consecuentemente se abordaban los temas históricos y de las antigüedades arquitectónicas y artísticas (arqueología en el concepto moderno). Por otro lado, estaban las publicaciones de viajes, en los cuales también se destacaban estos elementos patrimoniales. 
XVIII — con predominancia en las ciencias naturales y la mineralogía—. Por resultado de los estudios en cuanto al contexto, se evidenciaban hallazgos de antiguallas,${ }^{6}$ con mérito - en ese momento- a ser destacadas e investigadas apropiadamente, y luego difundidas en publicaciones exclusivas para instituciones y grupos científicos e intelectuales del mundo.

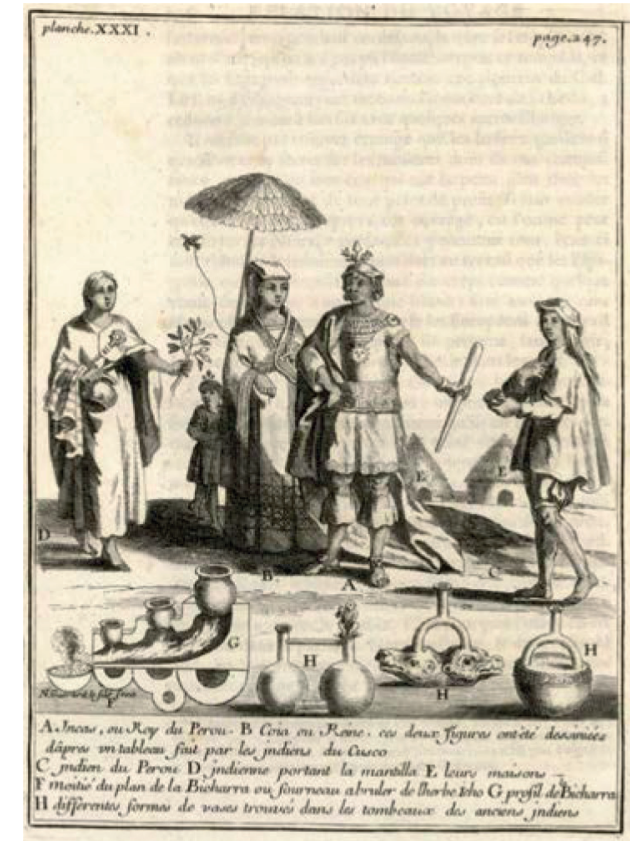

Figura 1. Objetos arqueológicos recopilados en el siglo XVIII Fuente: Petit Thouars (1840)

Nota: han sido difundidos como referentes patrimoniales de la historia y cultura prehispánica del Perú.

El segmento académico peruano —elitista para la época — estaba al corriente de aquellas investigaciones y fue parte de muchos de esos trabajos, ${ }^{7}$ promoviendo a partir de ello criterios de discusión sobre las corrientes de pensamiento patrimonialista, convergiendo luego en difusiones periódicas para el conocimiento social mayoritario. Estos aportes fueron impulsando con tenacidad a los movimientos independentistas (líderes y colectivos sociales), motivándolos a la reflexión y la valoración correspondiente respecto a la historia

6 Denominación de usanza colonial (especialmente por los cronistas) para referirse a un monumento o complejo arqueológico.

7 Como el caso de José Eusebio Llano Zapata, Hipólito Unanue y Mariano Eduardo de Rivero Uztariz, entre los siglos XVIII y XIX, respectivamente. 
peruana y a su producción cultural, intensificando la pretensión a reescribir la historia, con otro enfoque, pero en un escenario nuevo de nación libre. Para tales logros se crearon instituciones corporativas-culturales, las que impulsaron progresivamente a que esos argumentos de reivindicación histórica y patrimonial pasaran a ser soportes ideológicos, los cuales fueron planteados en los idearios políticos durante los primeros años de la independencia, teniendo como resultante el trazo de lineamientos de políticas culturales, ${ }^{8}$ luego establecidas y desarrolladas como políticas públicas.

En ese escenario reivindicativo y bajo aquellos criterios ideológicos, se gestaron nuevas formas de valor hacia el patrimonio cultural e histórico del Perú, las cuales se expandieron en el aprecio que iban desde nivel de la curiosidad hasta la construcción de un simbolismo individual y colectivo. Se convirtieron así paulatinamente en un elemento de significación plena del individuo, de su comunidad o de su nación, que — según la coyuntura— convergieron en procesos de identidad cultural.

\section{Ideario político y esbozos de políticas culturales}

El 10 de enero de 1822 se da la fundación de la entidad cultural denominada Sociedad Patriótica de Lima, que tiene la finalidad de crear una corriente de reflexión, análisis y discernimientos en materia cultural del Perú, especialmente en los temas de literatura, música, artes plásticas, ciencias naturales, historia y patrimonio, ${ }^{9}$ y cuyas resultantes y conclusiones de discusión eran difundidas con regularidad para el conocimiento de la dirigencia libertadora y la ciudadanía patriótica. Así mismo, la institución estaba facultada en sugerir y establecer los lineamientos para el desarrollo cultural de la nueva república, configurándose en un órgano ideológico — de apoyo - del naciente Estado independiente del Perú, cuyas directrices en el plano cultural eran influyentes y reconocidas por la autoridad gobernante.

8 Fueron planificaciones culturales visionarias para su momento, y las primeras en ser integradoras e inclusivas con la sociedad.

9 Sus campos de acción, según el Reglamento de la Sociedad Patriótica de Lima, publicado por el diario El Sol del Perú en 1822, se dividían en cuatro áreas: la filosofía política, las ciencias, la agricultura y la literatura. Esta última se vinculada al estudio de las bellas artes, en el cual se contemplaba —entre otros asuntos - lo concerniente a la historia y antigüedades del Perú. 
Categóricamente, la Sociedad Patriótica de Lima fue la institución que determinó las políticas culturales del momento ${ }^{10}$ e influyó en la creación de órganos culturales como el Museo Nacional y la Biblioteca Nacional. Así, tuvo la expectativa visionaria de propiciar conocimiento en la población para el cambio de pensamiento y la construcción de una nueva concepción sobre el entorno natural y el contexto social de la nación peruana y del mundo, instituida «con el fin de promover la ilustración, y discutir las materias que puedan influir en la mejora de nuestras instituciones» (Paredes 1822, 33).

El mismo protector general José de San Martín fue miembro honorario de ese organismo, quien destacó la prestigiosa labor que desempeñaba para el bienestar y prosperidad de los pueblos, siendo parte de los cambios y reformas que se daban en el país. La institución también tenía discreta participación en los aspectos políticos y económicos, cuyos miembros —intelectuales de más avanzada formación académica—, con la lucidez y entendimiento de su tiempo, enfocaron tenaz y gradualmente un sentimiento de patriotismo e identidad, basados en referentes culturales del país.

Los directivos de la Sociedad Patriótica de Lima establecieron estrategias de difusión, con planteamientos didácticos como medio de propaganda ${ }^{11}$ para enfatizar y garantizar los cambios políticos y, consecuentemente, los beneficios de sensibilidad ciudadana hacia los acervos culturales del país. Tenían la misión de colaborar para el conocimiento de los potenciales culturales de la nación, y en la toma de decisiones políticas de la nueva autoridad republicana.

El principal artilugio de difusión — en un primer momento - fue la publicación de la (el tabloide) Gaceta del Gobierno, con dos ediciones por semana. Allí se daban a conocer los manifiestos conclusivos más relevantes de interés coyuntural, proporcionadas por la Sociedad Patriótica de Lima. Además, se difundían los comentarios sobre las referidas informaciones y también se divulgaban los preceptos jurídicos provenientes e inspirados en los idearios políticos e ideológicos de dicha sociedad. Otro de los medios de propaganda en un nivel más académico fue el semanario informativo El Sol del Perú, en el cual se plasmaba abundante información reflexiva, ensayos, artículos y debates concernientes a la literatura, las artes, la historia y a las ciencias del Perú y del mundo, desde su primera salida el 14 de marzo de1822. Hubo

10 Porque eran parte de sus objetivos, como institución cultural y científica.

11 Emulando a la agrupación Sociedad Amantes del País de fines del siglo XVII en cuanto al criterio doctrinario, con la diferencia que los antecesores difundían sus hallazgos y discusiones en un círculo académico elitista, mientras que la Sociedad Patriótica de Lima tenía una expectativa y cobertura con mayor receptividad, siendo en sí una propaganda de adoctrinamiento. 
otros medios de propaganda libertadora, como el diario El Americano y Los Andes Libres, cuyos objetivos estaban centrados en dar noticias de los sucesos de campaña, y publicar arengas políticas provenientes de todo el territorio nacional para enarbolar el optimismo del ideal de cambio. Solo en ciertas oportunidades — si el contexto lo ameritaba — se hacía eco de los discursos de la Sociedad Patriótica de Lima, y en ocasiones se replicaban argumentos vinculados a la historia y cultura del país.

Las informaciones difundidas por tales medios fueron tarea de mucho rigor académico y compromiso político, ideológico y patriótico, que en su momento tuvieron receptividad, como «idea fuerza» en la progresión y configuración del nuevo país libre: ${ }^{12}$

Ya brilla ó [sic] humea la sagrada antorcha de la libertada en el nuevo mundo; y las letras siguen siempre de auxiliares á [sic] esa divinidad [...] asi [sic] la creación de esta sociedad científica conducida por el Richeliu de América, al tanto que la magnífica obra de la Biblioteca y Museo Nacional, y la importante reforma del Teatro, emprendido y casi ejecutado con una inesperada prontitud en medio de la guerra, y de la falta de recursos; son señales combinadas y bien expresivas, de que las letras van á fijar su domicilio en nuestro territorio, y que un jénio [sic] sublime les ha preparado ya templos y sacerdotes [...] transfigurándose a la América el esplendor de las ciencias [...] Las artes hermanas de las letras, remontarán el vuelo que han empezado á emprender [...] (San Donás $1822,5-6)$

\subsection{Planteamientos de políticas culturales}

En esa etapa de afianzar una nueva forma de Estado, los planes, las políticas y las gestiones de cambio fueron influenciados por las directrices de la Sociedad Patriótica de Lima, especialmente en lo cultural, creando las condiciones para una apertura y conectividad con lo histórico y con los recursos culturales de la nación, bajo el ideal de construir una identidad representativa. ${ }^{13}$ Se plantearon interpretaciones de la historia peruana, destacando las peculiaridades de las

12 Los ensayos, reflexiones, manifiestos y comentarios en los aspectos históricos estaban orientados a la reivindicación de los recursos culturales y de los acontecimientos relevantes que se vinculaban con las civilizaciones precolombinas, con una clara orientación de fomento y afirmación de una identidad cultural colectiva.

13 Con la clara y perseverante intención de fortalecer la independencia del país y garantizar su perdurabilidad. 
civilizaciones prehispánicas, llegando a la sublimación de los alcances tecnológicos, arquitectónicos y artísticos de la cultura inca.

Bajo esos códigos identitarios, se consolidaron los respaldos jurídicos, desde disposiciones y decretos hasta la primera Constitución del Perú en el periodo republicano (Pareja 1954), cuya asamblea constituyente se instaló en setiembre de 1822 y se aprobó en el primer tercio de 1823. Esto dio viabilidad a la creación de instituciones para el fomento y la gestión educativa, cultural y científica, cuyas aplicaciones se evidenciaron implementándose o reformándose los establecimientos educativos, acondicionándose espacios de apoyo al conocimiento humanístico y fundándose — principalmente — la Biblioteca Nacional y el Museo Nacional como primera línea de inclusión social hacia el conocimiento y la ilustración de la mayoría de los peruanos. ${ }^{14}$

La reflexión sobre la historia, la cultura y la ciencia, a consecuencia del fomento e información, alentó el interés de repensar sobre el legado cultural. Y desde las políticas públicas del nuevo Estado republicano se concibieron otros enfoques sobre los recursos culturales del Perú, principalmente en el patrimonio cultural, con valoraciones colectivas coincidentes, destacándose los hallazgos más relevantes de los estudios anteriores e intensificándose el interés por la investigación, como el caso del científico peruano Mariano Eduardo de Rivero Uztariz. ${ }^{15}$

Los cambios sociopolíticos avivaron un sentimiento social hacia el rescate y la protección de los recursos culturales. Esta postura fue fortalecida con medidas y políticas acertadas para promover expectativas y dar continuidad a la interpretación del patrimonio cultural.

El repensar en la historia y en los recursos culturales del Perú propició nuevas formas de valor simbólico y valor de uso, denominado «renovación y recordación histórica», ${ }^{16}$ impulsándose disposiciones de carácter oficial para los cambios en materia de reivindicaciones histórico-culturales. Así se inicia-

14 Porque el principio fundamental de la Sociedad Patriótica de Lima y del Gobierno de turno fue inferir en lo pedagógico, garantizar la enseñanza — especialmente de las ciencias, humanidades y artes - e impartir conocimiento pleno de la historia y cultura peruana, para forjar ciudadanos con visiones de desarrollo hacia la nueva patria.

15 Personaje erudito, natural de Arequipa, con trayectoria académica en Europa. Fue director del Museo Nacional en su segunda fundación de 1826. Fomentó el valor formal y el valor simbólico del patrimonio cultural del país, motivando a su salvaguarda como recurso histórico-cultural. Dedicó exploraciones e investigaciones sobre las ciencias naturales, la historia y los monumentos arqueológicos del Perú, teniendo entre sus obras relevantes Antigüedades peruanas, trabajada junto con el Dr. Jhon Diego Tschudi y publicada en Viena en 1851; así mismo, la Colección de memorias cientificas, publicada en Bruselas en 1857 y que recoge artículos publicados en la década de la independencia.

En la dimensión de la memoria cultural y no del chovinismo. 
ron nuevos reconocimientos de los símbolos históricos, los cuales fueron sustituidos en sus denominaciones y aspectos correspondientes, de acuerdo con los valores de uso que irrumpían en el periodo de transformación. Con ello se propiciaron nuevos significados y hubo identificación por parte de las poblaciones. Los efectos de esta reforma cultural se evidenciaron en los primeros meses después de la proclama de independencia, en varias ciudades y pueblos del Perú, intensificándose tales cambios en los años posteriores, con apoyo y beneplácito de las comunidades. Destacaron los cambios de nombres de determinadas ciudades y espacios abiertos, así como la categorización urbana de «pueblo a ciudad», tal como refiere el estudio sobre los diversos decretos de ese periodo, editados por la Universidad de la Plata (1950).

Las recomposiciones de valores simbólicos y valores de uso de la historia y de los espacios emblemáticos respondían a la postura ideológica y a las políticas culturales del Gobierno republicano peruano, en su etapa de composición y afianzamiento de una nueva nación. Del mismo modo, se desplegaron disposiciones para el cuidado, rescate y consolidación de los recursos culturales y naturales, para su aprecio y valoración mediante la enseñanza y la creación de espacios para tal fin.

\section{Recuerdo}

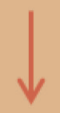

\section{Reconocimiento}

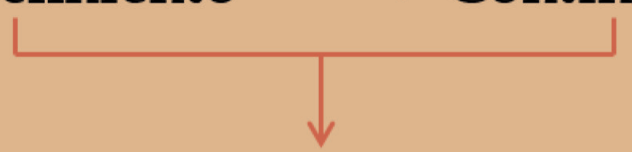

\section{Continuidad}

\section{Aprehensión}

Figura 2. Esquema de flujo para concebir la memoria histórica mediante la reivindicación Cultural.

Fuente: elaboración propia

Nota: recurso aplicado en el primer quinquenio de la independencia peruana para forjar una identidad a partir de la historia y la cultura originaria. 
Las políticas de reforma cultural sirvieron para el proceso de conocimiento de la historia peruana y su patrimonio monumental, con mayor inclusión colectiva, desde las instituciones educativas convencionales y con la creación de otros espacios públicos, como las instalaciones de recursos bibliotecarios en el país. Esto se inició con la fundación de la Biblioteca Nacional en la capital. Se establecieron así lineamientos con el fin de incentivar a la sociedad hacia un aprendizaje — en mayor escala — sobre la historia y cultura peruana, con el objetivo de construir un nuevo marco de identidad social. Para ello, era necesario un acercamiento al legado histórico-cultural, afirmándose en la memoria de la sociedad, lo cual se fue consolidando en un proceso escalonado de conexión y valoración.

La gobernanza en el primer quinquenio de la época república consideraba que la prosperidad de los peruanos tenía que enfocarse en forjar ciudadanos ilustrados. Para ello, la importancia de la labor educativa y el derecho al conocimiento mayoritario de los ciudadanos era fundamental. Dicho principio, por un lado, confrontaba a la educación elitista del sistema colonial, impuesta por más de tres siglos, y que postró a la mayor parte de los peruanos en la ignorancia y en el sometimiento, y casi en su totalidad a la clase indígena. Con ese ideario se resarcía el derecho de acceder al conocimiento y a la educación — meritorio para todo peruano-, con la voluntad de que recuperen su dignidad humana, más aún en un modelo social republicano.

Entre los resultados de mayor trascendencia, producto de esas medidas de políticas culturales, se fundaron las instituciones científico-culturales como la Biblioteca Nacional y el Museo Nacional, concebidas para brindar conocimiento y construir valores en la población.

La creación de la Biblioteca Nacional fue impulsada bajo una nueva forma de conciencia y razonamiento, para el beneficio ciudadano en función de sus derechos civiles. Con ello se hizo necesaria la acción de impartir conocimiento, ciencia y cultura, como parte de las políticas públicas, para dignificar y educar a la nueva sociedad peruana ${ }^{17}$ en aras de fortalecer la «libertad». Por ello, el protector José de San Martín — a un mes de la jura de independencia peruana - consideraba que la educación, el conocimiento y la cultura eran

17 Dirigido especialmente a la juventud, no solo con servicios y fomento para la lectura, sino con programas de capacitaciones, vinculadas a las áreas anexas. Este ha sido el caso de la Escuela de Humanidades, fundada en 1825, conocida también como Museo Latino, porque se impartían enseñanzas de idiomas, especialmente el latín; así mismo, se daban clases de filosofía y humanidades. 
«armas» para vencer la dominación y abrir camino al desarrollo, fundamentando en el marco del decreto para la creación de la Biblioteca Nacional, el cual proclamaba:

Facilitarles todos los medios de acresentar [sic] el caudal de sus luces, y fomentar su civilización, por medio de establecimientos útiles, es el deber de toda administración ilustrada. Las almas reciben entonces nuebo [sic] temple, toma vuelo [sic] el ingenio, nacen las ciencias [...] triunfan las leyes y la tolerancia, y empuña el cetro la filosofía, principio de toda libertad, consoladora de todos los males, y origen de todas las acciones nobles.

[...] las letras, y las ciencias ejercen sobre la prosperidad de un estado. (Gonzales $1825,19)$

A un año de la proyección de la Biblioteca Nacional, una vez implementada y acondicionada en su logística y en los fondos bibliográficos, se dispuso el Reglamento de Funcionamiento y la proyección cultural correspondiente el 31 de agosto de 1822. Se reafirmó con ello el objetivo de la institución en una sociedad libre y el valor de uso que proyectaba como servicio para el desarrollo humano y social del momento:

[...] bajo el sistema español [...] se han escaseado por una funesta política todos los recursos de la ilustración, prohibiendo la lectura de libros selectos y el estudio de las ciencias relativas á los derechos del hombre, un gobierno independiente debió facilitar desde sus primeros pasos, la adquisición de conocimientos útiles á todas las clases del estado. Con este objeto se resolvió la construcción de la Biblioteca nacional, hallándose ya concluida con bastante perfección y hermosura [...] (Gonzales 1825, 169-170).

Por otra parte, la reorganización de los centros educativos existentes y la creación de otros fueron canalizados hacia la inclusión social, lo que determinó su trascendencia como parte de las políticas culturales. La nueva república, con las medidas paulatinas en políticas culturales, fue consolidando el compromiso ciudadano con la historia y con su acervo cultural, creándose un acercamiento continuo en dos categorías sociales de ese periodo:

i) La construcción de la categoría de memoria cultural.

ii) La construcción de la categoría de identidad colectiva. 


\section{Fundación del Museo Nacional (1822, 1826): factores y efectos}

En la Gaceta del Gobierno, publicado el 3 de marzo de 1822, se anunciaba sobre las donaciones de fondos bibliográficos hechas por algunos ciudadanos - incluyendo al general San Martín - para la Biblioteca Nacional, en vísperas de su apertura. Se exhortaba al público a sumarse a dicha acción, y se invitaba a extender la misma iniciativa para el Museo Nacional. En ese comunicado, se hacía referencia sobre los espacios destinados a dichas instituciones, indicando que se encontraban en proceso de implementación. Ese comentario fue la primera mención documentada del proceso de creación del Museo Nacional, que — para esa fecha - estaba en etapa de acondicionamiento de sus instalaciones expositivas, administrativas y de almacenamiento para el acopio de colecciones museísticas. Por ello, en la nota informativa se persuade a los ciudadanos a la solidaridad con donaciones de objetos apropiados para el museo:

Esperamos que estos ejemplos sean imitados por los demás ciudadanos amantes del bien público, que se interesen en enriquecer, tanto la Biblioteca como el Museo Nacional, cuyo establecimiento ha emprendido el gobierno con la eficacia que a todos consta, y con intenciones que no pueden frustrarse, si todos coadyuvan a ellas en la parte que les toca (S/a 1822, 1)

Con el llamado a la donación de objetos patrimoniales a beneficio del «museo», se demuestra que dicho establecimiento cultural fue planificado para ser construido en el mismo inmueble destinado a la Biblioteca Nacional — que se encontraba en ejecución-. Esta aseveración es refrendada en el documento oficial de la Gaceta del Gobierno, el cual indicaba que el Museo Nacional estaba planeando implementarse «en el mismo edificio destinado a la biblioteca [...]» (Supremo Delegado 1822, 1-2). Es decir, en los ambientes del claustro desocupado por el Colegio de La Libertad, ${ }^{18}$ perteneciente al antiguo convento jesuita del Colegio Máximo de San Pablo, en la calle Los Estudios.

Deseando el gobierno establecer un museo nacional en el mismo edificio destinado a la biblioteca, no duda que todos los ciudadanos amantes de la honra de su país, contribuirán a enriquecerlo con cuantos objetos posean dignos por su

18 Esto ocurrió porque dicho colegio se trasladó a los ambientes del Convictorio de San Carlos. 
rareza, de servir de base a las preciosas colecciones que pueden formarse en el Perú de los tres reinos de la naturaleza, con ventajas que no son comunes a las otras partes del mundo. Los venerables restos que nos han quedado de las artes que poseían los súbditos del antiguo imperio de los Incas, merecen reunirse en aquel establecimiento, antes que acaben de ser exportados fuera de nuestro territorio, como lo han sido hasta aquí, porque era un interés de la España, el borrar todos los vestigios de la antigua civilización y grandeza. Las pinturas clásicas, estatuas ó bustos, colecciones de monedas, y cuanto pertenece a tan distinguido objeto, será admirado con gratitud, ó pagado su valor de los fondos aplicados a la instrucción pública.

Mientras se acaba de preparar el edificio, cuya obra se halla notablemente adelantada, los patriotas que quieran ceder libros ó alguno de los artículos indicados, pasarán una nota de ellos al ministerio de estado, reteniéndolos en su poder, hasta que sea tiempo de recogerlos.

Realizando este y otros proyectos, haremos ver de lo que es capaz un pueblo que tiene en sus manos su destino (Supremo Delegado 1822, 1-2).

A consecuencia de las políticas culturales del Gobierno en el periodo de transición hacia un Estado republicano, se determinó la fundación del Museo Nacional, institución con fines patrióticos para el desarrollo social de los peruanos en el ámbito científico y educativo. Este debía tener funciones claras para su desempeño, destacándose — a juicio nuestro - cinco aspectos esenciales para considerar al Museo Nacional como tal: entidad, salvaguarda, identidad, educación y memoria histórico-cultural, que lo caracterizaron por ser influyente en esa etapa.

En la condición de «entidad», el museo (en sus diferentes espacios de funcionamiento de esa época, como en el inmueble del Colegio la Libertad y en el edificio de la otrora Inquisición) también fue un componente cultural urbano relevante en el nivel de equipamiento a inicios del periodo republicano. Respecto al estado de «salvaguarda», el principal objetivo de la creación del primer museo en el Perú fue cautelar los bienes patrimoniales nacionales para impedir su comercialización ilegal y dar un freno al saqueo y tráfico de objetos arqueológicos y artísticos del territorio nacional. Se plantearon así disposiciones jurídicas y mecanismos reglamentarios para la salvaguarda correspondiente, facultándose además la penalización al expolio patrimonial y acreditándose el decomiso de los bienes a beneficio de la colección del Museo Nacional. Uno de estos decretos refiere el contexto de saqueo sistemático al que fueron expuestos los 
bienes culturales, lo que generaba una devastación del patrimonio arqueológico e impedía el conocimiento de dichos legados. Por ello, en un primer momento, el Gobierno del protectorado de San Martín determinó su salvaguarda y las sanciones respectivas, proyectándose la instalación del Museo Nacional como alternativa de protección y como un derecho cultural del ciudadano.

Los monumentos que quedan de la ántiguedad [sic] del Perú, son una propiedad de la nación, porque pertenecen a la gloria que deriva de ellos: las preciosidades de que abundan nuestros minerales, aunque puedan circular libremente en el país y mudar de dominio, pero el gobierno tiene un derecho a prohibir su exportación, cuando felizmente ha llegado el tiempo de aplicar a un uso nacional todo lo que nuestro suelo produzca de exquisito en los tres reinos de la naturaleza, Con dolor se han visto hasta aquí vender objetos inapreciables, y llevarse a donde es conocido su valor, privándonos de la ventaja de poseer lo nuestro. En precaucion [sic] de esto, se ha resuelto lo que sigue.

El SUPREMO DELEGADO. He acordado y decreto.

$1^{\circ}$. Se prohíbe absolutamente la extracción de piedras minerales, obras antiguas de alfarería, tejidos y demás objetos que se encuentren en las HUACAS, sin expresa y especial licencia del gobierno, dada con alguna mira de utilidad pública. $2^{\circ}$. El que contraviniere al artículo anterior, incurrirá en las pena [sic] de perdimiento de la especie, sea poco ó mucho su valor, la que se aplicará al museo nacional y mas [sic] mil pesos de multa aplicados a los fondos destinados a la instrucción pública. Los administradores de aduana y comandantes de resguardo, quedan encargados de velar la observancia de este decreto bajo su responsabilidad (Torre Tagle 1950 [1822], 1-2).

Del mismo modo, el compromiso de «identidad» se percibía favorable, al establecerse el contacto con los elementos patrimoniales que resguardaban el museo. Este fue un proceso que se iba generando por la consolidación de valores dados por la ciudadanía — según su interpretación - y por la progresiva identificación entre «pasado y presente». De esta manera se orientaba paralelamente al vasto conocimiento de su significación para así favorecer al proceso de «educación» en las mejores condiciones, cuya consecuencia más elevada fue el fomento de la «memoria histórica y cultural». ${ }^{19}$

19 Aunque en los primeros años de la independencia se incentivó, acertadamente, a un acercamiento con la historia y el patrimonio, posteriormente dicha conexión se fue debilitando, por las coyunturas sociales y políticas, hasta retomarse con el mismo impulso en los albores del centenario de la independencia. 
Esos ejes fueron las ventajas de la prosperidad museística en cuanto a su proyección social, generadas en un ambiente de cambios políticos, reinterpretaciones históricas y replanteamientos identitarios, que se fueron plasmando desde la primera fundación del Museo Nacional en 1822, pero luego fue interrumpida en lo que eran sus funciones de difusión y acopio - a causa de los rebrotes bélicos esporádicos-, durante el proceso de consolidación de la independencia del Perú. Estos eventos causaron zozobra en la población, con daños colaterales en los acondicionamientos de los edificios públicos de la ciudad capital, como el caso de las instalaciones del Museo Nacional, quedando inactiva hasta su segunda y definitiva fundación en $1826 .{ }^{20}$ En ese momento, la dirección del museo fue encargada — acertadamente - al científico, naturalista y visionario Mariano Eduardo Rivero Ustariz. Se retomaron, a partir de su administración, las propuestas museísticas en relación a su contexto patrimonial, con más despliegue y facilidad:

Queriendo el Consejo de Gobierno fomentar la enseñanza de las ciencias exactas, ha creído necesario a el logro de sus honrosos designios establecer el muséo [sic], proyectado en el año de 1822, para proporcionar a la juventud, que se dedique al estudio sublime de la naturaleza, colecciones escogidas que la instruyan en las propiedades de los seres orgánicos é inorgánicos.

[...] el gobierno se ha propuesto colocar los establecimientos públicos al nivel que se encuentran en las naciones civilizadas: y esta resolución, calculada para acelerar los progresos de la ilustración, le inspira la confianza de que todos los amantes del país de desprenderán generosamente de las rarezas naturales que posean, donandolas [sic] para el servicio y ornamento de tan importante institución. 5. Los tejidos y preciosidades estraidas [sic] de las huacas [...] se remitirán a los prefectos, para que las dirijan en primera oportunidad al director del muséo [sic] D. Mariano Rivero [...] (Serra 1826, 1)

En esa etapa, el establecimiento del Museo Nacional fue creado como entidad científica, dando continuidad a lo iniciado en 1822, pero intensificando su calidad. Y para ello tuvo utilidad la difusión de la resolución aludida, como información y sensibilización social para el propósito de rehabilitar una nueva propuesta museística, conforme al contexto y a las exigencias técnicas del mo-

20 El Ministerio de Gobierno y Relaciones Exteriores emitió el 8 de abril de 1826 una circular de carácter resolutiva, la cual contenía un manifiesto sobre la refundación del Museo Nacional y exhortaba a las autoridades regionales y locales para la salvaguarda del patrimonio cultural y natural. 
mento, predominando la función científica del Museo Nacional y destacando la participación ciudadana para su implementación y fomento adecuado.

Los cambios políticos en los primeros cinco años de la independencia peruana desarrollaron planes y acciones en el manejo de la gestión y la actividad cultural que — desde un análisis actual— fueron «políticas culturales» apropiadas, especialmente porque se dio en un contexto social agitado y cuyos antecedentes en cuanto a la «memoria cultural colectiva» eran displicentes —en el sector mayoritario- hacia los legados patrimoniales e históricos. En esa situación, las políticas culturales se tornaron paulatinamente en responsabilidades para la evocación de la historia y de los elementos que lo representaban, intensificándose el espíritu de patriotismo en una coyuntura que lo ameritaba. Este proceso se fue dando en la medida que los idearios políticos de la administración gubernamental de turno se expandían y persuadían, fortaleciéndose con nuevos criterios e instrumentos de poder, instaurados en ambos periodos de la gestión de los libertadores José de San Martín y Simón Bolívar, para garantizar y dar continuidad a los derechos culturales de los ciudadanos, entre los cuales destacaba la institución del Museo Nacional.

En esta etapa de transformación cultural, se destacan cuatro elementos que - de acuerdo con nuestro análisis — fueron líneas de políticas culturales como:

i) La contribución para valorar el legado patrimonial.

ii) Destacar los recursos simbólicos.

iii) Construir un horizonte de memoria y sentido histórico.

iv) Garantizar la continuidad histórica y cultural.

Con el primer lineamiento, se fue generando un acercamiento, valoración y respeto de la población — entre ellos el sector académico - hacia las edificaciones prehispánicas. Se produjo así un auge de motivación e interés por la investigación y estudio de dichos recursos. Así mismo, las contribuciones y donativos de objetos museísticos fueron incrementando la colección del Museo Nacional.

La eficiencia de las gestiones culturales vinculadas al Museo Nacional y al patrimonio histórico influyeron en otras iniciativas del ramo, con implementaciones de espacios para la difusión y conservación artística mediante exposiciones de obras de arte y actividades musicales. Ese fue el caso del decreto con fecha del 2 de diciembre de 1825, emitido por el Consejo de Gobierno y firmado por sus miembros: Hipólito Unánue, Juan Salazar y José de Larréa 
y Loredo, por encargo del ministro de Gobierno Lino Ruíz de Pancorbo. Así, se ordenó la adjudicación de un local apropiado con la finalidad de promover las actividades artísticas de la Sociedad Filarmónica y la creación del Museo de Pintura. ${ }^{21}$ Se concedió para tal fin varios espacios del edificio de la antigua Inquisición, correspondiéndole a la Filarmónica la capilla, algunos salones y el patio, y dos salones para el Museo de Pintura.

EL CONSEJO DE GOBIERNO [...] no se ha creído menos obligado a contribuir a los progresos de las nuevas artes, cuando de su general propagación depende, en gran parte, la perfección del estado social. La música y la pintura, entre ellas, merecen sin duda un lugar preferente; $y$ aunque la primera sea la más adelantada en esta capital, no pasa la segunda del grado de mediocridad á que quedó reducida en el régimen colonial. [...] DECRETA:

1. ${ }^{\circ}$ Se concede a la sociedad filarmónica, para los objetos de un instituto, la capilla de la inquisición, con tres piezas laterales y un patio pequeño dependiente de estas.

2. ${ }^{\circ}$ No se empleará el edificio principal en otro destino que el de conciertos de música, debiendo colocarse, en la mayor parte de su recinto, asientos de firme para los espectadores.

4. ${ }^{\circ}$ Los dos salones siguientes de la misma inquisición se dedican a un Muséo [sic] de pintura, y las habitaciones del centro con las demás piezas del patio principal, para la habitación del Director que fuere de este ramo.

5. ${ }^{\circ}$ Los cuadros y pinturas mas [sic] esquisitas [sic] de la propiedad del Estado serán reunidas en los espresados [sic] salones (Consejo de Gobierno 1967 [1825], art, 1, 2, 4 y 5).

21 Eran instituciones relevantes en la difusión de la música académica y de las artes pláticas, en cuyas proyecciones sociales destacaban el aporte al conocimiento y a la apreciación estética del arte musical y pictórico, inculcándose una sensibilización hacia ellas. A pesar de que la intensión política del momento fue masificar esas instituciones artísticas, no tuvo mucho éxito en la inclusión social. 
TABLA 1

Conclusiones de cuadros de memorias

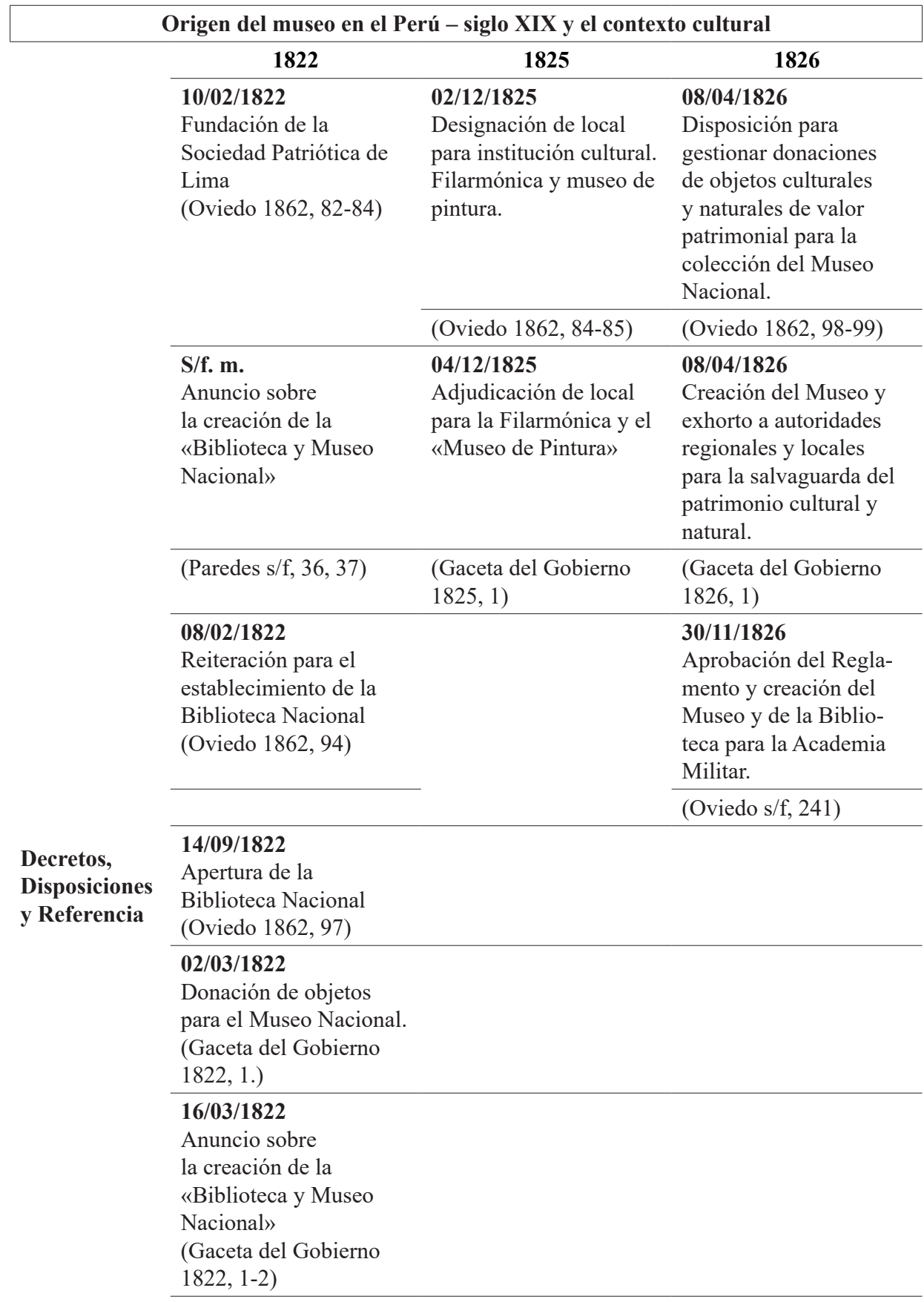




\section{3/04/1822}

Exhortación a

la salvaguarda y

conservación del

patrimonio cultural

mueble e inmueble.

(Gaceta del Gobierno

1822, 1)

03/04/1822

\begin{tabular}{l}
\hline Salvaguarda del \\
\hline patrimonio cultural y \\
\hline natural (emitido el \\
\hline $02 / 04 / 1822)$.
\end{tabular}

(Gaceta del Gobierno

1822, 1)

Nota. Esquema de gestión de políticas culturales en el contexto de la creación del Museo Nacional durante el primer quinquenio de la independencia.

Elaboración propia.

Por tanto, consideramos que esos logros fueron iniciativas de una «gestión en políticas culturales» de la época para establecer equipamientos culturales, con el objetivo de desarrollar la difusión, conservación y valoración de las artes en un nuevo escenario social de república, por la importancia y repercusión que tenía dicha acción en el desarrollo y dignidad de la sociedad.

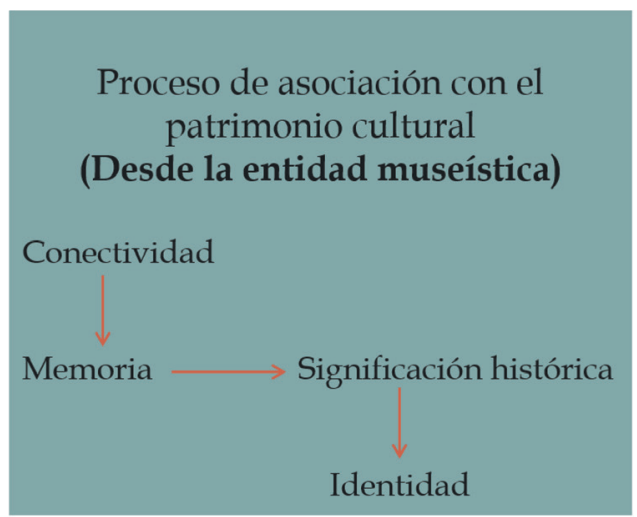

Figura 3. Esquema de flujo del rol del museo en el proceso identitario Fuente: elaboración propia

El segundo lineamiento establecía los elementos de valor simbólico, referenciados en las categorías históricas y estéticas que se daban a conocer 
mediante la difusión del arte, el patrimonio edificado prehispánico y la historicidad del Perú antiguo, proyectando estructurar una identificación colectiva. Con esas pautas, se posibilitaba cimentar la «memoria cultural» mediante el descubrimiento de la significación en el referente histórico. Esos procesos fortalecían la preservación de la cultura, historia e identidad, con lo cual se destacaban las propuestas de la tercera y cuarta línea de acción, vinculadas a la memoria y a la continuidad histórica-cultural, respectivamente.

El resultado más emblemático, producto de las políticas culturales del primer quinquenio de la independencia del Perú, fue la creación del Museo Nacional, el que - a pesar de los intentos de conexión con la comunidad y la inclusión para su acceso - apenas tuvo la accesibilidad de una minoría, especialmente del sector académico. Sin embargo, el «museo» trascendió como símbolo de cultura, patrimonio e historia, erigiéndose categóricamente como base de la institución museística del Perú, porque las fundaciones posteriores del principal «museo» del país se dieron sobre la configuración del primigenio Museo Nacional, reestableciéndose e incrementando su colección, reacondicionándose y adecuando los espacios de funcionamiento. Así, más que todo se fue refundando su institucionalidad en diversos períodos, y readecuando su rol de funciones; todo ello de acuerdo con las coyunturas político-culturales en la historia del país.

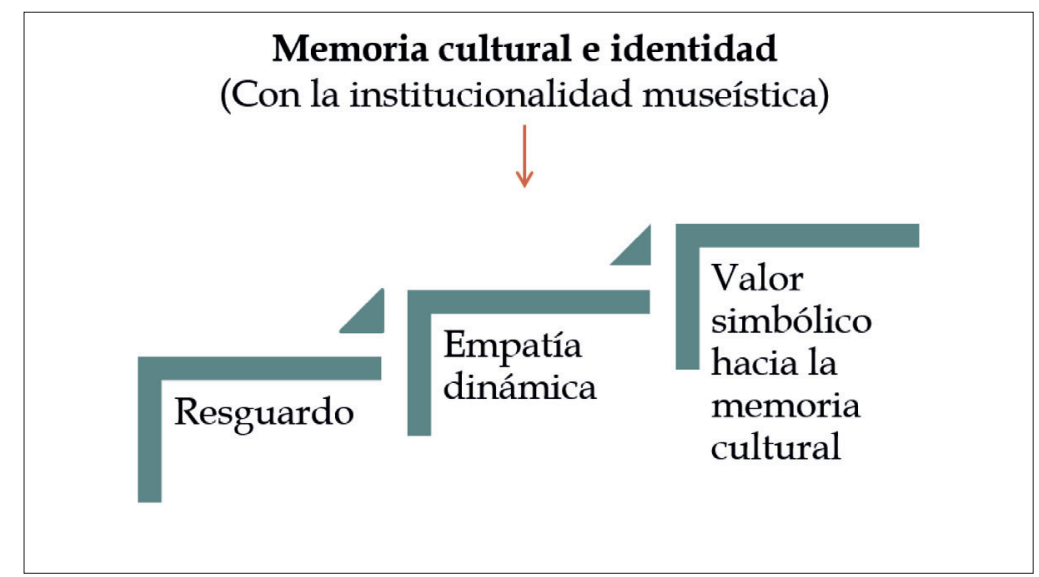

Figura 4. Efectos del museo en la memoria cultural y la identidad Fuente: elaboración propia

Los efectos en el mismo contexto fueron las creaciones de distintos espacios culturales y académicos que influenciaron en la reflexión continua de lo histórico y lo cultural del Perú. Así, se dio una actitud colectiva de «repensar 


\section{en lo cultural», que benefició al compromiso del ideario de la nueva república y al afán por consolidarla.}

\section{TABLA 2}

Contraste de la fundación del Museo Nacional entre 1822 y 1826

\begin{tabular}{|c|c|c|}
\hline \multicolumn{3}{|c|}{ Características y contexto del Museo Nacional } \\
\hline & 1822 & 1826 \\
\hline 1 & $\begin{array}{l}\text { Se proyecta su creación junto con la } \\
\text { Biblioteca Nacional. }\end{array}$ & Consolidación, reapertura y refundación. \\
\hline 2 & $\begin{array}{l}\text { Por Decreto Supremo N, }{ }^{\circ} 38 \text {, del } 8 \text { de } \\
\text { febrero, dado por el Supremo Delegado, } \\
\text { se instala la infraestructura del mes de } \\
\text { febrero al mes de setiembre, en las aulas } \\
\text { del antiguo Colegio de la Libertad, junto } \\
\text { a la Biblioteca Nacional. }\end{array}$ & $\begin{array}{l}\text { Se instala en los espacios del antiguo Tribunal } \\
\text { de la Inquisición. }\end{array}$ \\
\hline 3 & $\begin{array}{l}\text { Se encarga la dirección a Bernardo } \\
\text { Monteagudo (ministro de Estado, } \\
\text { Gobierno y Relaciones Exteriores). }\end{array}$ & $\begin{array}{l}\text { Mediante circular del Ministerio de Gobierno } \\
\text { y Relaciones Exteriores, del } 8 \text { de abril, firmado } \\
\text { por José Antonio Serra, se exhorta a la donación } \\
\text { y adquisiciones de objetos patrimoniales para el } \\
\text { fondo museográfico. }\end{array}$ \\
\hline 4 & $\begin{array}{l}\text { Su organización e implementación se } \\
\text { da por Decreto Supremo. } 02 / 4 / 1822, \\
\text { firmado por Torre Tagle, con el objetivo } \\
\text { de coleccionar y salvaguardar el } \\
\text { patrimonio arqueológico y natural. }\end{array}$ & $\begin{array}{l}\text { Se encarga la dDirección a Mariano Eduardo d } \\
\text { Rivero Uztariz }\end{array}$ \\
\hline 5 & $\begin{array}{l}\text { Tiene como objetivo la salvaguarda } \\
\text { de los bienes culturales para brindar } \\
\text { conocimiento, construir identidad y } \\
\text { memoria histórica. }\end{array}$ & $\begin{array}{l}\text { Funciona en el lugar hasta } 1830 \text {, fecha en que se } \\
\text { traslada a la capilla del mismo edificio. }\end{array}$ \\
\hline 6 & $\begin{array}{l}\text { Se exhorta a la ciudadanía para } \\
\text { donativos de fondos museográficos. }\end{array}$ & $\begin{array}{l}\text { En el contexto, hubo un sosiego político, el } \\
\text { cual determinó sentimientos más consolidados } \\
\text { hacia los recursos históricos y culturales, } \\
\text { a consecuencia del despliegue informativo } \\
\text { y la aplicación de las reformas de políticas } \\
\text { culturales, generando interés en la población } \\
\text { sobre el legado cultural y propiciando la } \\
\text { creación de otros organismos culturales. }\end{array}$ \\
\hline
\end{tabular}

7 El proceso de implementación, acopio y funcionamiento es interrumpido por la guerra.

8 Contextualmente, aún se percibía la convulsión política, y a pesar de ello se daban nuevos enfoques sobre los recursos culturales y fomento de la memoria histórica, mediante el acercamiento al legado histórico y cultural. En esta etapa, la Sociedad Patriótica de Lima tuvo mayor participación en las decisiones de las políticas culturales.

Nota. Cuadro comparativo del contexto y características de la fundación del Museo Nacional, en dos periodos trascendentes de la etapa de independencia peruana.

Fuente: Paredes (1822, 33-39), Carrasco, (1826, 84-85), Tello y Mejía (1967, 1-58)

Elaboración propia. 


\section{Conclusiones}

En los primeros cinco años de república independiente del Perú, se influyó en el proceso social para el reconocimiento de la memoria cultural mediante la difusión y gestión de políticas culturales efectivas, basadas en idearios políticos y sustentados con aplicaciones jurídicas. Esto condujo a resultados importantes como la creación del Museo Nacional, que contribuyó con la sociedad a construir valores simbólicos respecto a los objetos patrimoniales y al pasado prehispánico. Fueron sensibilidades, razonadas principalmente por los manifiestos e instrumentos jurídicos, cuyos tenores se enfocaban en el valor, la salvaguarda y el conocimiento del legado patrimonial e histórico.

El ideario, como base para las políticas culturales en el contexto de la independencia del Perú, proyectaba el conocimiento de los recursos patrimoniales, generando empatía de la sociedad con la memoria cultural. Por medio de la difusión de los legados culturales e históricos de los peruanos, se establecieron niveles de acercamientos e interpretaciones a los recursos patrimoniales, que progresivamente lograron construir identificaciones colectivas como «herencia simbólica». Gracias a la estrategia de las líneas de políticas culturales, se contribuyó a la formación de una «memoria cultural» posindependencia, con sus propios valores y perspectivas que han trascendido, y que en la actualidad forman parte de nuestra idiosincrasia y nos representa con las características que cada generación le va otorgando. Por lo tanto, es una «memoria» de historia, de cultura y de espacio, cuyo vinculo dinámico es el Museo Nacional, el cual, a pesar de la dicotomía entre la elite intelectual y la ciudadanía en general para el acceso museístico, tiene una función que sigue siendo relevante como institución científica-cultural. 


\section{Referencias bibliográficas}

Ballart, J. 2002. Pasado historia y patrimonio. En El patrimonio histórico y arqueológico: valor y uso (pp. 29-59). Barcelona: Ariel.

Bourdieu, P. 1990. Sociología y Cultura. Ciudad de México: Editorial Grijalbo.

Carrasco, E. 1826. Calendario y guía de forasteros de Lima. Para el año de 1826. Lima: Imprenta del Estado.

Consejo de Gobierno. 1967 (1825). Decreto. Gaceta del Gobierno, VIII(45), 197. Caracas: Fundación Eugenio Mendoza.

Dorbigny, M. A., y Eyries, J. V. (Eds.). 1842. Viaje pintoresco a las dos Américas, Asia y Árica (tomo II, lám. 6). Barcelona: Imprenta y Librería de Juan Olivares.

Fuentes, M. A. 1858. Estadística general de Lima. Lima: Tip. Nacional de M. N. Corpancho (por J. H. del Campo).

Gonzales, J. 1825. Colección de Leyes y Decretos sancionados desde la Jura de la Independencia (tomo 1). Lima: Imprenta del Estado.

ICOM-Consejo Internacional de Museos. 2007. Estatutos del ICOM. París: ICOM.

ICOM-Consejo Internacional de Museos. 2019. Definición de museo. La creación de una nueva definición de museo-la columna vertebral del ICOM. París: ICOM.

Mulder, F. J. S/f. Lima, Ciudad de los Reyes, Corte y Emporio del Imperio Peruano, murada. Según la Arquitectura Militar y planta moderna tiene de circulación nueve millas, en que no se comprehenden sus Arrabales [cartografía]. Madrid: Biblioteca Nacional de España.

Oviedo, J. 1863. Colección de Leyes, Decretos y Órdenes publicadas en el Perú desde el año de 1821 hasta el 31 de diciembre de 1859. En Fuentes, M. A. y Bailly, F. (Eds.). Lima: Ministerio de Beneficencia, Instrucción Pública y Justicia.

Paredes, José Gregorio. 1822. Guía de forasteros de Lima, corregido. Para el año de 1822. Lima: Imprenta del Estado.

Pareja Paz Soldán, J. 1954. Las Constituciones del Perú. Madrid: Ediciones Cultura Hispánica.

Serra, J. 1967 (1826). Circular a los Prefectos, Intendentes, Municipalidades y Párrocos. Gaceta del Gobierno, IX(29), 353. Caracas: Fundación Eugenio Mendoza.

San Donás, Conde de. 1822 (13 de junio). Discurso. El Sol del Perú, (9), 4-5.

Sociedad Patriótica de Lima. 1822 (25 de abril). Reglamento de la Sociedad. El Sol del Perú, (6), 4.

S/a. 1822. Comunicado. Gaceta del Gobierno, II(18), 1. Ciudad de la Plata: Ministerio de Educación, Universidad de la Plata. 
Supremo Delegado. 1950 (1822). Comunicado Oficial. Gaceta del Gobierno, II(22), 1-2. Ciudad de la Plata: Ministerio de Educación, Universidad de la Plata.

Tello, J. C. y Mejía Xesspe, M. T. 1967. Historia de los museos nacionales del Perú, 18221946. Arqueológicas, (10). Museo Nacional de Antropología y Arqueología.

Torre Tagle-Supremo Delegado. 1950 (1822). Comunicado Oficial y Decreto. Gaceta del Gobierno, II(27), 1-2. Ciudad de la Plata: Ministerio de Educación, Universidad de La Plata.

Tschudi, J. y Rivero, E. M. 1851. Antigüedades peruanas. Viena: Imprenta de la Corte y del Estado.

Universidad de la Plata. 1950. Gaceta del Gobierno de Lima independiente, I-III (julio 1821-diciembre 1822). Ciudad de la Plata: Ministerio de Educación. 